\title{
State of the Art Regarding the Management of Multiligamentous Injuries of the Knee
}

\author{
Nigel T. Mabvuure ${ }^{1}$, Marco Malahias², Behrooz Haddad ${ }^{4}$, Sandip Hindocha ${ }^{*, 3}$ and Wasim S. Khan ${ }^{4}$ \\ ${ }^{I}$ Brighton and Sussex Medical School, Brighton, BN2 5XL,UK \\ ${ }^{2}$ Department of Plastic Surgery, Countess of Chester Hospital, Chester, CH2 1UL, UK \\ ${ }^{3}$ Department of Plastic Surgery, Whiston Hospital, Merseyside, L35 5DR, UK \\ ${ }^{4}$ University College London Institute of Orthopaedics and Musculoskeletal Sciences, Royal National Orthopaedic \\ Hospital, Stanmore, Middlesex, HA7 4LP, UK
}

\begin{abstract}
Multiligamentous knee injuries are rare but serious injuries that can threaten limb viability. As such, they require careful management to give patients the best chance of immediate and ultimate functional recovery. However, as these injuries are rare, there is paucity in prospective comparative studies large enough to provide high level evidence for best practice. This lack of comprehensive and convincing evidence has made the management of multiligamentous knee injuries an area of active debate and controversy. The debate on whether surgical management leads to better outcomes than non-operative management, the optimal timing of surgery after injury and whether repair is better than reconstruction is still ongoing. Using the Oxford Levels of Evidence, this review summarises the latest high level evidence to provide answers to these issues. Recommendations for practice have also been offered and assigned a grade using a published scale.
\end{abstract}

Keywords: Best practice, knee injuries, ligaments, management, Oxford levels of evidence.

\section{INTRODUCTION}

Acute multiligamentous knee injuries (MKIs) are usually the results of high energy injuries that may dislocate the knee. They may also present as chronic injuries. These injuries are rare but they can potentially threaten limb viability as they can have associated neurovascular deficits. As such, they require careful treatment to give patients the best chance of immediate and ultimately a fully functional recovery. Goals of immediate treatment include the management of pain and any neurovascular deficits that may threaten life and limb. The goals of definitive management include restoring knee stability, full range of motion, managing long term pain and delivering patients to, at least, their pre-injury level of function or activity [1]. However, as these injuries are rare, there is a paucity of prospective comparative studies large enough to provide high-level evidence for best practice $[2,3]$. This lack of comprehensive and convincing evidence has made the management of MKIs an area of active debate and controversy [4]. The debate on whether surgical management leads to a better outcome than conservative management, the optimal timing of surgery since time of injury and whether repair is better than reconstruction are still ongoing. Using the Oxford Levels of Evidence (OLE) (Table 1), this review summarises the latest high level evidence to provide answers to these issues.

*Address correspondence to this author at the Department of Plastic Surgery, Whiston Hospital, Merseyside, L35 5DR, UK; Tel: 01244366265; Fax: 01244366266; E-mail: hindocha2001@yahoo.com
Table 1. Oxford level of evidence scale.

\begin{tabular}{|c|l|}
\hline Level & \multicolumn{1}{|c|}{ Evidence } \\
\hline \hline Level 1 & $\begin{array}{l}\text { Meta-analysis of randomised controlled trials (RCT) or } \\
\text { high quality RCTs }\end{array}$ \\
\hline Level 2 & $\begin{array}{l}\text { Lesser quality randomised control trials or prospective } \\
\text { comparative studies }\end{array}$ \\
\hline Level 3 & Case control studies or retrospective studies \\
\hline Level 4 & $\begin{array}{l}\text { Case series without the use of comparison or control } \\
\text { groups }\end{array}$ \\
\hline Level 5 & Case reports or expert opinion \\
\hline
\end{tabular}

One of the difficulties in MKI research is the various definitions adopted at different centres. Some authors include into this definition any injuries where at least two ligaments are disrupted, whereas others have suggested more specific criteria. These differences in classification introduce a difficulty in revealing the true epidemiology of MKIs. In this review, MKIs are defined as any injuries where more than one ligament of the knee is disrupted.

\section{LITERATURE SEARCH}

The EMBASE and MEDLINE databases were searched from inception to July 2012 using the National Health Service (NHS) Evidence interface (http://www.evidence. nhs.uk/). The following search term was used: 
Duplicate filtered: [("multiple knee ligament"

OR multiligament* OR multi-ligament*).ti,ab

[Limit to: English Language]].

Two authors independently screened all abstracts and article titles for relevance to the present review. Full text copies of all potentially relevant papers were obtained and examined by one author. Articles were included if they were systematic reviews or RCTs (either OLE 1 and 2) or if they were case control studies or retrospective studies providing a comparison (OLE 3). The included articles were rated on the quality of their evidence according to the Oxford Centre of Evidence scale (Table 1) and assigned a grade of recommendation according to a published scale (Table 2), as done by Lo et al. in a previous review [5]. Case series without the use of comparison or control groups (OLE 4) were excluded, as were case reports and expert opinion (OLE 5).

Table 2. Grades of recommendation assigned according to the level of the evidence and its applicability to a target population [6].

\begin{tabular}{|c|l|}
\hline Grade & \multicolumn{1}{|c|}{ Level of the Evidence } \\
\hline \hline Grade A & $\begin{array}{l}\text { Level 1 evidence that is directly applicable to the } \\
\text { target population }\end{array}$ \\
\hline Grade B & $\begin{array}{l}\text { Extrapolated level 1 evidence, or directly applicable } \\
\text { level 2 evidence }\end{array}$ \\
\hline Grade C & $\begin{array}{l}\text { Extrapolated level 2 evidence or directly applicable } \\
\text { level 3 or 4 evidence }\end{array}$ \\
\hline Grade D & All other evidence from case reports and expert opinion \\
\hline
\end{tabular}

To avoid duplication and systematic bias, only the latest articles providing the highest levels of evidence were included. For example, where there were numerous retrospective studies (OLE 3) that have all been included in a systematic review, only the review will be included as it provides the higher level of evidence (OLE 2).

\section{SURGICAL REPAIR VERSUS CONSERVATIVE MANAGEMENT}

All surgeries have inherent risks for the patient including infection and those associated with anaesthesia. The decision to operate is one that should be taken only after careful deliberation and offered to patients with clear benefits. Nonoperative management of MKIs usually involves external fixation or casting. Levy et al. performed a systematic review of all studies comparing operative and non-operative treatment of MKIs up to August 2007 [2]. This review included three retrospective cohort analyses [7-9] and a meta-analysis of studies performed up to the year 2000 [10]. Levy et al.'s systematic review amalgamated data from these four studies and found that patients receiving operative treatment returned to work earlier than those that where managed non-operatively ( $72 \%$ versus $52 \%$ ) [2]. Surgically treated patients could also return to playing sport earlier than their non-operatively managed counterparts $(29 \%$ versus $10 \%$ ). However, there was not much difference on posttreatment range of motion (ROM) between these two groups $\left(126^{\circ}\right.$ versus $\left.123^{\circ}\right)$; nor was there a difference in mean flexion loss. No statistical analysis was performed to ascertain the significance of any of these differences. Surgical treatment also resulted in higher Lysholm scores (80 versus 57) and International Knee Documentation Committee (IKDC) scores (58 versus 20). In these functional outcome scales [11], higher scores are ideal.

Lysholm knee scale is a patient-reported scale used for knee-ligament surgery follow-up. It has 8 subscales rating limp, support, stair climbing, squatting, instability, locking and catching, pain and swelling. Scores below 65 are poor, fair between 65-83, good between 84-94 and excellent from $95-100$. IKDC is an 18 question-based patient-reported scale from 0 (worst) to 100 (best). Tegner activity level scale is a patient-rated sport-specific activity level measure. Zero points denote disability secondary to knee problems, 1-5 denotes ability to work or participate in recreational sports and 6-9 denotes increasing recreational and competitive sporting, national or international level football.

Peskun and Whelan performed an evidence-based review of studies comparing operative and non-operative treatment in the decade up to 2011 [4]. A total of 31 articles met their criteria for inclusion, all of which included patients who had received operative management. Only four of these articles directly compared outcomes in patients who had received non-operative management, the majority of whom were treated more than 20 years ago. The operative cohorts had significantly higher Lysholm scores than the non-operative cohort $(84.3 \%$ versus $67.2 \%, \mathrm{p}<0.05)$. However, the differences between the IKDC and Tegner scores between the groups failed to reach statistical significance, although they were higher in the operative cohorts in both cases $(69.0$ versus 63.7 and 4.8 versus 2.7 respectively). No significant differences were seen in the post-operative ROMs and knee contracture rates $(\mathrm{p}>0.05)$. A significantly greater proportion of patients who underwent surgery returned to work than those who underwent non-operative management $(80.9 \%$ versus $57.8 \%, \mathrm{p}<0.05)$. Likewise, the average proportion of patients in the operative cohort returning to their pre-injury athletic activity was significantly greater than in the nonoperative cohort $(50.0 \%$ versus $22.2 \%, \mathrm{p}<0.05)$. These results of return to activity are concordant with those found by Levy et al., summarised above.

It appears from the above data that operative management has some benefits to patients who receive it. These patients are more likely to return to their pre-injury activity, making this an attractive treatment option for people with active lifestyles and occupations such as sports people. There were also modest improvements in some functional scores but this was inconsistent between the studies, although the Lysholm score was significantly higher in the operative cohorts in both reviews presented above and in the meta analysis by Dedmond and Almekinders [10]. However, the data presented provides, at most, OLE 2 due to the inclusion of a meta analysis. It could also be argued that due to the low quality of data presented, the evidence presented may even be OLE 3. For example, the overall quality of the studies included in Peksun and Whelan's systematic review was poor (3.0 on the Newstead-Ottawa scale) [4]. There is also great diversity in the management protocols of the patients in all the studies included in these reviews, limiting the strength of the evidence. 
It is important to note that although surgery appears to be associated with improved outcomes, significant disability is still possible, even after successful surgery [10]. In some cases, non-operative management remains the preferable option. These include the elderly, inactive patients with comorbid contraindications to surgery, poorly compliant patients as well as trauma patients $[4,12]$.

\section{Recommendation}

There is some evidence to suggest that operative management is superior to non-operative management, especially with regard to return to activity and function as measured by the Lysholm score.

Level of evidence: OLE $2 / 3$.

Grade of recommendation: Grade C.

\section{TIMING OF SURGERY}

There is ongoing debate regarding the best time to intervene surgically as there have been various studies supporting both early and late intervention. Both approaches have their benefits and drawbacks. Early surgery is said to allow better visualization of the intra-articular anatomy, improving the potential for successful direct repair of the injured structures, although it increases the risk of compartment syndrome due to arthroscopic fluid extravasation, secondary to capsular injury [12]. There may also be an increased incidence of post-operative motion loss [13]. Conversely, whilst delayed surgery decreases swelling and provides time for capsular and ligamentous healing, it may also result in scarring that necessitates ligamentous augmentation or reconstruction [12].

Mook et al. performed a systematic review of literature up to 2008, aiming to determine whether early or late surgery was associated with better outcomes [3]. This was the first systematic review to attempt an answer to this question. Early intervention was defined as treatment within three weeks of injury and interventions after three weeks were regarded as late or chronic treatment. They found 24 retrospective studies (evidence level III or IV) comparing early versus late surgical intervention in 396 knees. Patients treated acutely were more likely to suffer anterior instability (OR 2.58, $<<0.05)$ and lose flexion (OR 5.18, $<<0.05)$ than those treated late. There was no statistically significant difference between the groups in the rates of valgus laxity, average range of motion, rates of extension loss and Lysholm scores $(p>0.05)$. However, patients treated acutely tended to rate their functional outcomes better than those treated late.

The decision to intervene early or late should also take into account the patient's social and occupational history. Contact sportspeople may be particular susceptible to these high energy injuries and the time to their return to normal activities should be an important consideration. There was some evidence in Mook et al.'s paper suggesting that patients managed early or acutely, returned to work later than patients managed in a staged manner $(\mathrm{OR} 0.09, \mathrm{p}<0.05)$ [3]. Other considerations include the mechanism of injury. For example, in battlefield amputees, the high incidence of infection and soft tissue injury indicates delayed treatment [14].

The systematic review by Mook et al. provides the highest level evidence to date but it is beset by several limitations, all acknowledged by the authors [3]. This review did not include any prospective, randomised controlled trials but included retrospective studies with heterogeneous patient populations. Therefore, the authors did not make any treatment recommendations but concluded that delayed treatment may yield equivalent outcomes to acute treatment. We add to this that a patient's social, occupational history and mechanism of injury should also be considered before the decision is made. Levy et al. also note that some differences between the acute and chronic treatment groups may have confounded the results of these comparative studies [15]. For example, they suggest that perhaps chronic patients suffered worse outcomes because they might have had concomitant severe soft tissue trauma that caused a delay in their definitive surgical treatment. Confounders such as these may be limited by various techniques including randomized study designs: the difficulty of this approach with regards to MKIs has been discussed above.

Recommendation: There is insufficient evidence on which to base recommendations.

Level of evidence: OLE 3.

Grade of recommendation: N/A.

\section{REPAIR VERSUS RECONSTRUCTION}

In practice, allograft and/or autograft reconstruction has largely replaced ligament repair [15]. There have been a few studies comparing outcomes in patients undergoing either repair or reconstruction of ligaments injured as part of MKI. Mariani et al. retrospectively analysed the outcome in patients who had undergone direct cruciate ligaments repair $(\mathrm{n}=11)$, anterior cruciate ligament $(\mathrm{ACL})$ reconstruction and posterior cruciate ligament $(\mathrm{PCL})$ reattachment $(n=6)$ and PCL reconstruction with ipsilateral bone-patellar tendonbone and ACL reconstruction with doubled semitendinosus and gracilis tendons $(n=6)$ [16] These patients were followed up for an average of 6.9 years. Combined ACL and PCL reconstruction resulted in better stability and an improved range of motion as compared to direct repair and ligament reattachment. There was no significant difference in the Lysholm or IKDCC scores of these groups but direct repair of cruciate ligaments resulted in significantly higher rate of the sag sign and a lower rate of return to pre-injury activity levels. Importantly, this study showed that there were no significant functional differences between the two groups.

Levy et al. compared clinical outcomes in 10 patients who underwent repair of the fibular collateral ligament (FCL) and postero-lateral corner (PLC) followed by delayed cruciate reconstructions, with 18 patients who underwent one-stage reconstructions [17]. All patients had suffered injuries to multiple ligaments. The proportion of patients with a failed result was significantly higher in the group that had undergone repair $(40 \%$ versus $6 \%, \mathrm{p}<0.05)$. This results is concordant with Stannard et al.'s results that showed that outcomes were more likely to be regarded successful if a patient had undergone reconstruction (91\% versus $63 \%$, 
$\mathrm{p}<0.05)$ [18]. The groups did not show statistically significant different clinical measures of stability; mean IKDC and Lysholm scores and average range of motion at final follow-up. The authors, as a result of this study, now favour one-stage reconstructions of the FCL/PLC in MKIs [17].

There are other studies that did not meet the full inclusion criteria of the present review but merit mention. These include the prospective study by Stannard et al., referred to above, that compared the outcomes between 39 patients who had undergone repair of PLC knee structures with 25 who had primary reconstructions [18]. The results of this study were in line with Levy et al.'s [17]. However, the repair and reconstruction groups in this study included patients with both single and multiple ligament injuries. Also, patients were not randomised into the respective treatment groups but allocated, possibly introducing bias. This however, is also true of Levy et al.'s study, that was also limited by the heterogeneity of their study group; another possible confounding factor may be that the study groups were not matched [17].

Recommendation: Considering that the only consistent finding was that repair is more likely to fail and that the rest of the outcomes were not significantly different, we recommend that reconstruction be considered, where there are no contraindications.

Level of evidence: 3 .

Grade of recommendation: Grade C.

\section{CONCLUSION}

There remains a paucity of high quality and high evidence level studies on which recommendations on the management of multiligamentous injuries may be based upon. The current evidence has mainly been pooled from multiple case series and retrospective cohort analyses of poor quality.

National [15] and international collaboration is encouraged to design better studies although this may be hindered by different management protocols in different localities. This will allow the development of stronger recommendations for practice. We also recommend that authors, where possible, adopt the Schenck classification system of knee dislocations to standardise reporting [19].

\section{CONFLICT OF INTEREST}

The authors confirm that this article content has no conflict of interest.

\section{ACKNOWLEDGEMENTS}

Declared none.

\section{REFERENCES}

[1] Cole BJ, Harner CD. The multiple ligament injured knee. Clin Sports Med 1999; 18(1): 241-62.

[2] Levy BA, Dajani KA, Whelan DB, et al.. Decision Making in the Multiligament-Injured Knee: An Evidence-Based Systematic Review. Arthroscopy 2009; 25(4): 430-8.

[3] Mook WR, Miller MD, Diduch DR, Hertel J, Boachie-Adjei Y, Hart JM. Multiple-ligament knee injuries: a systematic review of the timing of operative intervention and postoperative rehabilitation. J Bone Joint Surg Am 2009; 91(12): 2946-57.

[4] Peskun CJ, Whelan DB. Outcomes of operative and nonoperative treatment of multiligament knee injuries: an evidence-based review. Sports Med Arthrose Rev 2011; 19(2): 167-73.

[5] Lo S, Hallam MJ, Smith S, Cubison T. The tertiary management of pretibial lacerations. J Plast Reconstr Aesthet Surg 2012; 65(9): 1143-50

[6] Harbour R, Miller J. A new system for grading recommendations in evidence based guidelines. BMJ 2001; 323(7308): 334-6.

[7] Wong CH, Tan JL, Chang HC, Khin LW, Low CO. Knee dislocations-a retrospective study comparing operative versus closed immobilization treatment outcomes. Knee Surg Sports Traumatol Arthrosc 2004; 12(6): 540-4.

[8] Richter M, Bosch U, Wippermann B, Hofmann A, Krettek C. Comparison of surgical repair or reconstruction of the cruciate ligaments versus nonsurgical treatment in patients with traumatic knee dislocations. Am J Sports Med 2002; 30(5): 718-27.

[9] Rios A, Villa A, Fahandezh H, de Jose C, Vaquero J. Results after treatment of traumatic knee dislocations: a report of 26 cases. J Trauma 2003; 55(3): 489-94.

[10] Dedmond BT, Almekinders LC. Operative versus nonoperative treatment of knee dislocations: a meta-analysis. Am J Knee Surg 2001; 14(1): 33-8.

[11] Wright RW. Knee sports injury outcome measures. J Knee Surg 2005; 18(1): 69-72.

[12] Cox CL, Spindler KP. Multiligamentous knee injuries - surgical treatment algorithm. N Am J Sports Phys Ther 2008; 3(4): 198203.

[13] Bottoni CR, Liddell TR, Trainor TJ, Freccero DM, Lindell KK. Postoperative range of motion following anterior cruciate ligament reconstruction using autograft hamstrings: a prospective, randomized clinical trial of early versus delayed reconstructions. Am J Sports Med 2008; 36(4): 656-62.

[14] Kilcoyne K, Dickens J, Kroski W, Waterman S, Davila J. Ligamentous knee injuries in amputees. J Surg Orthop Adv 2012; 21(1): 44-9.

[15] Levy BA, Fanelli GC, Whelan DB, et al.. Controversies in the treatment of knee dislocations and multiligament reconstruction. J Am Acad Orthop Surg 2009; 17(4): 197-206.

[16] Mariani PP, Santoriello P, Iannone S, Condello V, Adriani E. Comparison of surgical treatments for knee dislocation. Am J Knee Surg 1999; 12(4): 214-21.

[17] Levy BA, Dajani KA, Morgan JA, Shah JP, Dahm DL, Stuart MJ. Repair versus reconstruction of the fibular collateral ligament and posterolateral corner in the multiligament-injured knee. Am J Sports Med 2010; 38(4): 804-9.

[18] Stannard JP, Brown SL, Farris RC, McGwin G, Jr., Volgas DA. The posterolateral corner of the knee: repair versus reconstruction. Am J Sports Med 2005; 33(6): 881-8.

[19] Schenck Jr R. Classification of knee dislocations. Oper Tech Sports Med 2003; 11(3): 193-8. 